\title{
MicroRNA-103 suppresses glioma cell proliferation and invasion by targeting the brain-derived neurotrophic factor
}

\author{
LING WANG $^{1}$, YING LIU ${ }^{2}$ and JIANWEI SONG ${ }^{1}$ \\ Departments of ${ }^{1}$ Neurosurgery and ${ }^{2}$ Emergency Surgery, Linyi People's Hospital, Linyi, Shandong 276000, P.R. China
}

Received March 28, 2017; Accepted September 6, 2017

DOI: $10.3892 / \mathrm{mmr} .2017 .8282$

\begin{abstract}
Glioma is the most common and aggressive of malignant brain tumours. MicroRNAs (miRNAs/miRs) are involved in tumour development of various human cancers, including glioma. Therefore, miRNAs may have potential tumour diagnostic, prognostic and therapeutic values in human glioma. miR-103 is abnormally expressed in various human cancer types. However, the detailed expression pattern, biological functions and underlying molecular mechanism of miR-103 in glioma remain unclear. Therefore, the present study aimed to investigate the expression, biological roles and underlying mechanisms of miR-103 in glioma. Results of the present study demonstrated that miR-103 was significantly down-regulated in glioma tissues and cell lines. Functional experiments demonstrated that miR-103 overexpression inhibited the proliferation and invasion of glioma cells in vitro. Additionally, brain-derived neurotrophic factor (BDNF) was identified as a direct functional target of miR-103 in glioma. Furthermore, mRNA and protein expression levels of BDNF were highly upregulated in glioma tissues compared with normal brain tissues. Spearman's correlation analysis indicated a negative association between miR-103 and BDNF mRNA expression levels in glioma tissues. Furthermore, rescue experiments demonstrated that BDNF up-regulation reversed the suppressive effects of miR-103 on glioma cell proliferation and invasion. Therefore, the authors of the present study hypothesized that the interaction between miR-103 and BDNF serves a role in glioma progression and, in the future, may serve as a therapeutic target for glioma treatment.
\end{abstract}

\section{Introduction}

Glioma is the most common and aggressive of malignant the brain tumours, and accounts for $\sim 30 \%$ of all central nervous

Correspondence to: Professor Jianwei Song, Department of Neurosurgery, Linyi People's Hospital, 49 Yizhou Road, Linyi, Shandong 276000, P.R. China

E-mail: jwsong_linyi@sina.com

Key words: brain-derived neurotrophic factor, microRNA-103, glioma, proliferation, invasion system tumours and $80 \%$ of all malignant brain tumours (1). Based on their pathology, gliomas can be divided into astrocytomas, glioblastomas, medulloblastomas, ependymomas and oligodendrogliomas (2). To date, the molecular mechanisms underlying the occurrence and development of gliomas remain unclear. Gliomas can be associated with several risk factors, including tumour origin, genetic factors, biochemical environment, ionizing radiation, nitroso compounds, air pollution, unhealthy lifestyle habits and infection $(3,4)$. At present, surgery combined with radiotherapy, chemotherapy and targeted therapy is the standard treatment for patients with glioma (5). Despite an evident progress in the therapeutic strategies for gliomas, the prognosis for patients afflicted with this disease remains poor (6). The median survival time of patients with glioma is $~ 9-12$ months (7). The poor prognosis of patients with glioma is partially due to the highly invasive nature of glioma cells, making complete surgical resection unattainable $(8,9)$. Therefore, elucidation of the mechanisms underlying the formation and progression of glioma and development of effective diagnostic and therapeutic treatment methods for patients with glioma are required.

MicroRNAs (miRNAs/miRs) are a class of endogenous, short noncoding 19-25 nucleotides-long RNA molecules (10). miRNAs negatively modulate gene expression at the transcriptional level by base pairing with the 3'-untranslated region (3'-UTRs) of their target mRNAs, and inducing mRNA degradation or inhibition of translation (11). Through negative regulation of the target genes, miRNAs participate in many physiological processes, including cell proliferation, cell cycle progression, differentiation, apoptosis and metastasis (12-15). Recent studies have reported that miRNAs are abnormally expressed in human cancers, including glioma, breast, gastric, bladder and lung cancers (16-20). In particular, abnormal expression of certain miRNAs is associated with tumorigenesis and cancer progression $(21,22)$. Dysregulated miRNAs may serve similar roles to oncogenes or tumour suppressor genes, depending on their target mRNAs (23). Therefore, miRNAs may demonstrate a potential as novel prognostic indicators and therapeutic targets for patients with malignancies (24).

miR-103 is abnormally expressed in various human cancers (25-27). The present study aimed to elucidate the expression pattern, biological functions and underlying molecular mechanism of miR-103 in glioma. 


\section{Materials and methods}

Tissue samples and cell lines. The present study was approved by the Ethical Committee of Linyi People's Hospital (Linyi, China). Written informed consent was obtained from all participants. A total of 26 surgically resected glioma tissues were collected from patients with glioma (male, 25; female, 11; age range, 37-72 years) in Linyi People's Hospital between July 2013 and February 2016. A total of 12 normal brain tissue samples were obtained from patients with cerebral trauma (male, 7; female, 5; age range, 25-68 years) subjected to a surgical procedure. None of the patients were treated with radiotherapy or chemotherapy prior to the surgical resection. All collected tissues were immediately snap-frozen in liquid nitrogen at the time of surgery and stored at $-80^{\circ} \mathrm{C}$.

Normal human astrocytes (NHAs) were purchased form ScienCell Research Laboratories, Inc. (San Diego, CA, USA) and cultured in the astrocyte medium (ScienCell Research Laboratories, Inc.). Glioma cell lines U251, LN18 and LN229 were obtained from the Cell Bank Type Culture Collection of the Chinese Academy of Sciences (Shanghai, China). All glioma cell lines were maintained in Dulbecco's modified Eagle medium (DMEM) containing 10\% fetal bovine serum (FBS), $100 \mathrm{U} / \mathrm{ml}$ penicillin and $100 \mathrm{mg} / \mathrm{ml}$ streptomycin (all Invitrogen; Thermo Fisher Scientific, Inc., Waltham, MA, USA) at $37^{\circ} \mathrm{C}$ in a humidified atmosphere containing $5 \% \mathrm{CO}_{2}$.

Cell transfection. miR-103 mimics and negative control miRNA (miR-NC) were purchased from Guangzhou RiboBio Co., Ltd. (Guangzhou, China). Brain-derived neurotrophic factor (BDNF) overexpression plasmid (pcDNA3.1-BDNF) and blank plasmid (pcDNA3.1) were obtained from Shanghai GenePharma Co., Ltd. (Shanghai, China). For transfection, cells were seeded in 6-well plates at a density of $1 \times 10^{5}$ cells/per well and cultured until 60-70\% confluence was attained. Cells were separately treated with pcDNA3.1-BDNF or pcDNA3.1 using Lipofectamine 2000 (Invitrogen; Thermo Fisher Scientific, Inc.,), according to the manufacturer's protocol. Following incubation at $37^{\circ} \mathrm{C}$ with $5 \% \mathrm{CO}_{2}$ for $8 \mathrm{~h}$, the transfection mixture was replaced with fresh DMEM supplemented with 10\% FBS, without penicillin and streptomycin. Following transfection for $24 \mathrm{~h}$, MTT assay was performed. Reverse transcription-quantitative polymerase chain reaction (RT-qPCR) and Transwell invasion assays were performed at $48 \mathrm{~h}$ post-transfection. Western blotting analysis was performed to detect protein expression at $72 \mathrm{~h}$ post-transfection.

$R T-q P C R$. Total RNA was extracted from tissues or cells using the TRIzol reagent (Thermo Fisher Scientific, Inc.), according to the manufacturer's protocol. To quantify the expression of miR-103, total RNA was reverse transcribed into cDNA by using the TaqMan MicroRNA Reverse Transcription kit (Applied Biosystems; Thermo Fisher Scientific, Inc.). The temperature protocol for reverse transcription was as follows: $16^{\circ} \mathrm{C}$ for $30 \mathrm{~min}, 42^{\circ} \mathrm{C}$ for $30 \mathrm{~min}$ and $85^{\circ} \mathrm{C}$ for $5 \mathrm{~min}$. Subsequently, the TaqMan MicroRNA PCR kit (Applied Biosystems; Thermo Fisher Scientific, Inc.) was used to detect miR-103 expression in an RT-qPCR amplification reaction, with U6 as an internal reference gene. To quantify the BDNF mRNA expression, cDNA was synthesized using the M-MLV
Reverse Transcriptase (Promega Corporation, Madison, WI, USA), followed by a qPCR reaction with the SYBR Green Real-Time PCR Master Mix (Toyobo Life Science, Osaka, Japan) using a 7500 Sequence Detection system (Applied Biosystems; Thermo Fisher Scientific, Inc.), with GAPDH as an internal control gene. The thermocycling conditions for qPCR were as follows: $95^{\circ} \mathrm{C}$ for $10 \mathrm{~min}$, followed by 40 cycles of $95^{\circ} \mathrm{C}$ for $15 \mathrm{sec}$ and $60^{\circ} \mathrm{C}$ for $1 \mathrm{~min}$. The following PCR primer pairs were designed: miR-103 forward: 5'-GAGCAG CATTGTACAG-3', reverse: 5'-GTGCAGGGTCCGAGG T-3'; U6 forward: 5'-CTCGCTTCGGCAGCACA-3', reverse: 5'-AACGCTTCACGAATTTGCGT-3'; BDNF forward: 5'-TCCCTGGCTGACACTTTT-3', reverse: 5'-ATTGGGTAG TTCGGCATT-3'; GAPDH forward: 5'-CGGAGTCAACGG ATTTGGTCGTAT-3', reverse: 5'-AGCCTTCTCCATGGT GGTGAAGAC-3'. Relative gene expression was calculated using the $2^{-\Delta \Delta C \mathrm{C}}$ method (28).

MTT assay. MTT assay (Sigma-Aldrich, Merck KGaA, Darmstadt, Germany) was used to determine cell proliferation. Cells were seeded in 96-well plates at a density of $3 \times 10^{3}$ cells/well and cultured overnight at $37^{\circ} \mathrm{C}$ with $5 \% \mathrm{CO}_{2}$. Subsequently, transfection was performed, as described above, and the samples were incubated at $37^{\circ} \mathrm{C}$ with $5 \% \mathrm{CO}_{2}$ for 0,24 , 48 and $72 \mathrm{~h}$. At each time point, $10 \mu 1$ MTT solution $(5 \mathrm{mg} / \mathrm{ml})$ was added into each well and incubated for additional $4 \mathrm{~h}$, and cells were then lysed in $150 \mu \mathrm{l}$ dimethylsulfoxide at room temperature for $10 \mathrm{~min}$. The absorbance was measured at a wavelength of $490 \mathrm{~nm}$ with a SpectraMax M3 microplate reader (Molecular Devices, LLC, Sunnyvale, CA, USA). Each assay was performed in triplicate and repeated independently at least three times.

Transwell invasion assay. Transwell filters (pore size, $8 \mu \mathrm{m}$; EMD Millipore, Billerica, MA, USA) coated with Matrigel (BD Biosciences, Franklin Lakes, NJ, CA, USA) were used to examine cell invasion ability. Cells were harvested $48 \mathrm{~h}$ post-transfection and $5 \times 10^{4}$ cells were resuspended in 200- $\mu$ 1 FBS-free DMEM and placed in the upper chamber. A total of $500 \mu 1$ DMEM containing 20\% FBS was added into the lower chamber as a chemo-attractant. Cells were then incubated at $37^{\circ} \mathrm{C}$ with $5 \% \mathrm{CO}_{2}$ for $24 \mathrm{~h}$. Cells on the upper surface of the membrane were removed with cotton swabs. Cells that invaded to the bottom of the insert were fixed in $4 \%$ paraformaldehyde at room temperature for $10 \mathrm{~min}$, stained with $0.1 \%$ crystal violet at room temperature for $10 \mathrm{~min}$ and washed in PBS. Finally, the invasive cells were counted under a light microscope (Olympus IX53; Olympus Corporation, Tokyo, Japan) in five independent fields/well (magnification, x200).

Bioinformatics analysis. TargetScan Human online software (version 7.0; www.targetscan.org) and miRBase database (http://www.mirbase.org/) were used to predict the targets of miR-103.

Luciferase reporter assay. For the luciferase reporter assay, a reporter plasmid containing the wild-type (pmirGLO-BDNF-3'-UTR Wt) or mutant (pmirGLO-BDNF-3'-UTR Mut) 3'-UTR of BDNF were synthesized by Shanghai GenePharma Co., Ltd. Cells were seeded in 

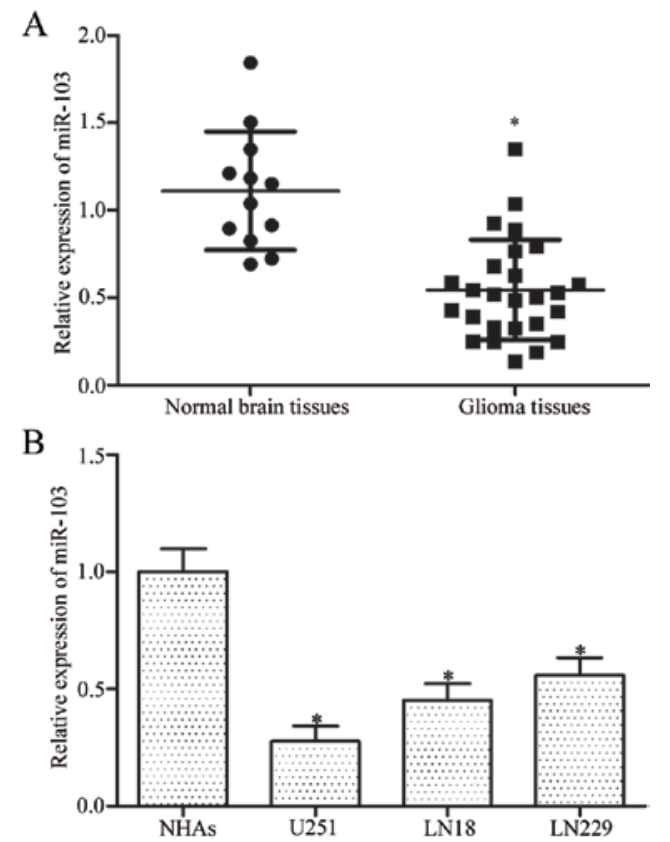

Figure 1. miR-103 is down-regulated in glioma tissues and cell lines. (A) miR-103 expression in glioma tissues $(\mathrm{n}=26)$ and normal brain tissues $(n=12)$ was examined by reverse transcription-quantitative polymerase chain reaction. (B) Relative expression of miR-103 was determined in three human glioma cell lines (U251, LN18 and LN229) and in NHAs. Data are presented as the mean \pm standard deviation. ${ }^{*} \mathrm{P}<0.05$ vs. the respective control group. miR-103, microRNA-103; NHAs, normal human astrocytes.

24 well plates at a density for $1.5 \times 10^{5}$ cells per well and incubated overnight at $37^{\circ} \mathrm{C}$ with $5 \% \mathrm{CO}_{2}$. Cells were then transfected with miRNA mimics (50 pmol; miR-103 mimics or miR-NC) and reporter plasmids $(0.2 \mu \mathrm{g}$; pmirGLO-BDNF-3'-UTR Wt or pmirGLO-BDNF-3'-UTR Mut) using Lipofectamine 2000, according to the manufacturer's protocol. Following a $24 \mathrm{~h}$ transfection, the cells were harvested and luciferase activities were determined using the Dual-Luciferase Reporter Assay system (Promega Corporation) according to the manufacturer's protocol. The Renilla luciferase activity was normalized to the Firefly luciferase activity. This assay was performed in triplicate and repeated three times independently.

Protein isolation and western blot analysis. Proteins were extracted from tissue samples or cells using a protein lysis buffer (Xinyu Biotechnology, Shanghai, China) supplemented with a protease and phosphatase inhibitor cocktail (Roche Diagnostics, Basel, Switzerland). A Bicinchoninic Acid Protein Assay kit (Pierce; Thermo Fisher Scientific, Inc.) was applied to measure protein concentration. Equal quantities of proteins $(30 \mu \mathrm{g})$ were analyzed by $10 \%$ SDS-PAGE and transferred onto polyvinylidene fluoride membranes (EMD Millipore). The membranes were further blocked with $5 \%$ skimmed milk at room temperature for $2 \mathrm{~h}$ and incubated overnight at $4^{\circ} \mathrm{C}$ with monoclonal primary antibodies: Anti-mouse anti-human BDNF (cat. no. ab10505; 1:1,000 dilution; Abcam, Cambridge, UK) or anti-mouse anti-human GAPDH (cat. no. sc-47724; 1:1,000 dilution; Santa Cruz Biotechnology, Inc., Dallas, TX, USA). Subsequently, the membranes were incubated with a corresponding horseradish peroxidase-conjugated secondary antibody (cat. no. c-2005; 1:5,000 dilution; Santa Cruz
A

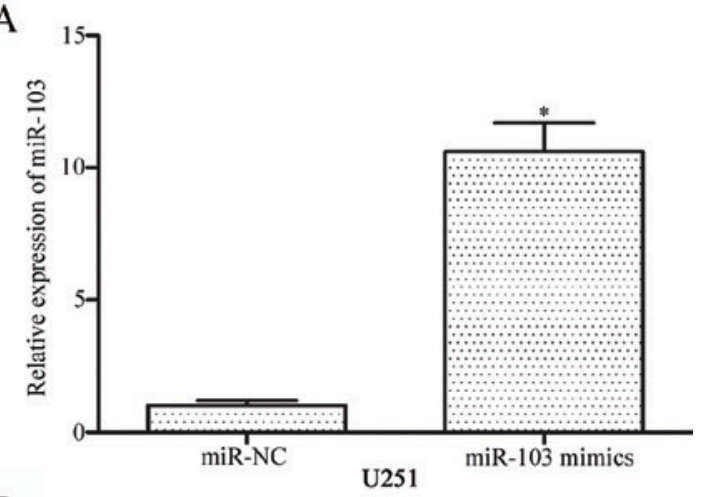

B

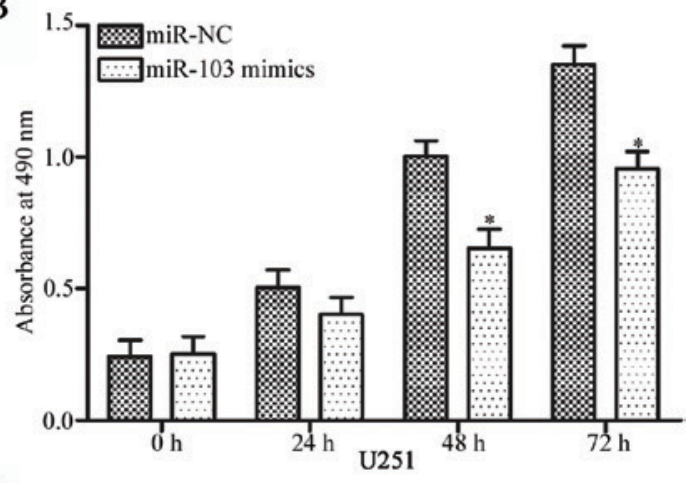

$\mathrm{C}$

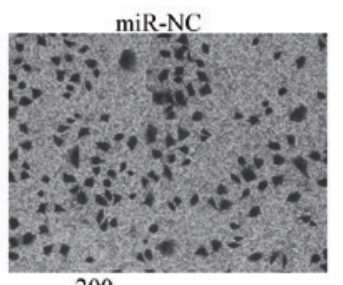

miR-103 mimics
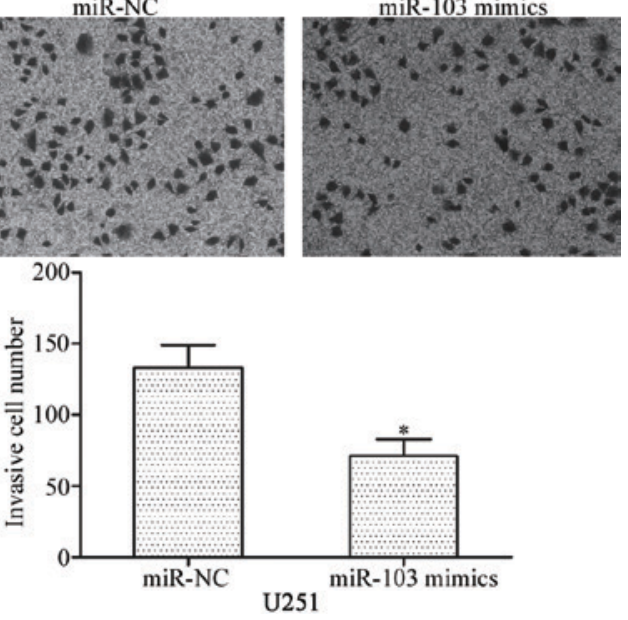

Figure 2. miR-103 overexpression inhibits the in vitro proliferation and invasion of U251 cells. (A) U251 cells were treated with miR-103 mimics or miR-NC. Transfection efficiency was determined by reverse transcription-quantitative polymerase chain reaction. (B) MTT assay demonstrated that cell proliferation was inhibited by miR-103 overexpresssion in U251 cells. (C) Transwell invasion assay demonstrated that cell invasion capabilities were also suppressed by miR-103 in U251 cells. Data are presented as the mean \pm standard deviation. ${ }^{*}<0.05$ vs. the respective control. MiR-103, micoroRNA-103; NC, negative control.

Biotechnology, Inc.) at room temperature for $2 \mathrm{~h}$. Protein blots were detected using the Enhanced Chemifluorescence western blotting kit (Pierce; Thermo Fisher Scientific, Inc.). Signal intensity was quantified using ImageJ software (version 1.48; National Institutes of Health, Bethesda, MA, USA). GAPDH was used as control.

Statistical analysis. Data are presented as the mean \pm standard deviation. Comparisons between groups were made with Student's t-tests or one way analysis of variance using SPSS 
A

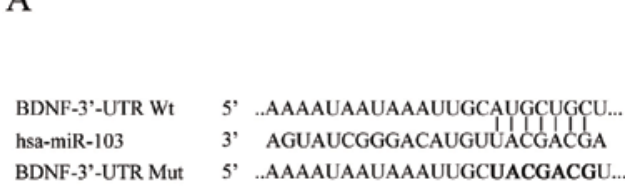

$\mathrm{C}$

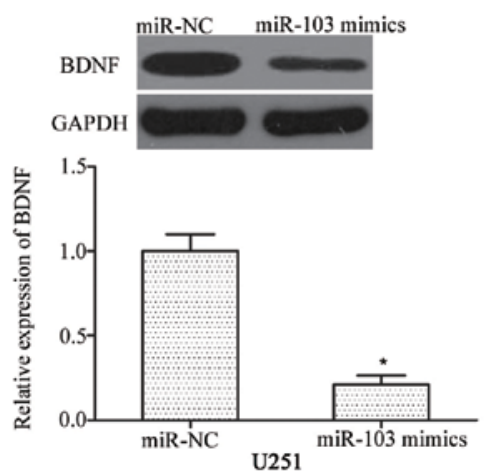

B

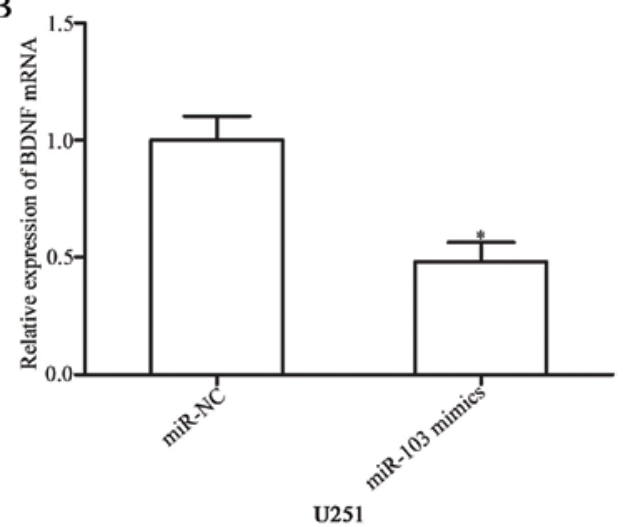

D

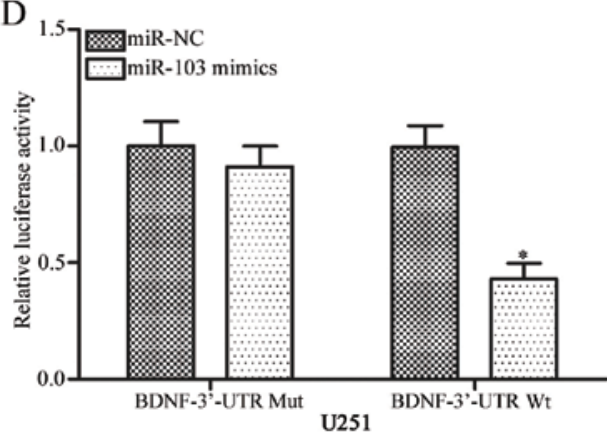

Figure 3. BDNF is a direct target of miR-103 in glioma. (A) Visualization of a potentially complementary region between the seed region sequence of miR-103 and nucleotide 299-305 region in the 3'-UTR of BDNF, determined using bioinformatics tools. (B) mRNA and (C) protein expression of BDNF in U251 cells transfected with miR-103 mimics or miR-NC. (D) Luciferase reporter assays were performed in U251 cells following co-transfection with pmirGLO-BDNF-3'-UTR Wt or pmirGLO-BDNF-3'-UTR Mut and miR-103 mimics or miR-NC. Data are presented as the mean \pm standard deviation. ${ }^{*}$ P $<0.05$ vs. the respective control. miR-103, micoroRNA-103; BDNF, brain-derived neurotrophic factor; Wt, wild-type; Mut, mutant; NC, negative control; UTR, untranslated region.

software (version 18.0, SPSS, Inc., Chicago, IL, USA). Student-Newman-Keuls analysis was used to compare differences between groups. $\mathrm{P}<0.05$ was considered to indicate a statistically significant difference.

\section{Results}

miR-103 is significantly down-regulated in glioma tissues and cell lines. The expression level of miR-103 in 26 glioma tissues and 12 normal brain tissues was measured using RT-qPCR to assess the potential role of miR-103 in glioma. Results demonstrated that miR-103 was down-regulated in glioma tissues compared with the normal brain tissues (Fig. $1 \mathrm{~A}, \mathrm{P}<0.05$ ). Furthermore, miR-103 expression was detected using RT-qPCR in three glioma cell lines (U251, LN18 and LN229) and NHAs. The expression level of miR-103 decreased in glioma cell lines compared with NHAs (Fig. 1B, P<0.05). The above data support the hypothesis that miR-103 is down-regulated in glioma tissues and cell lines, suggesting that miR-103 downregulation may be involved in glioma initiation and progression.

miR-103 inhibits the proliferation and invasion of glioma cells in vitro. To investigate the roles of miR-103 in glioma, U251 cells were transfected with miR-103 mimics to increase the intracellular levels of miR-103. RT-qPCR confirmed that miR-103 expression was markedly up-regulated in U251 cells transfected with miR-103 mimics compared with cells transfected with miR-NC (Fig. 2A, P<0.05).
MTT assay was performed to investigate the effect of miR-103 overexpression on glioma cell proliferation. As presented in Fig. 2B, miR-103 up-regulation inhibited the proliferation of U251 cells compared with miR-NC-transfected cells $(\mathrm{P}<0.05)$. Transwell invasion assay was further used to examine the invasive capability of glioblastoma cells. Ectopic expression of miR-103 significantly decreased the invasive capacities of U251 cells (Fig. $2 \mathrm{C}, \mathrm{P}<0.05$ ). These results of the present study indicate that miR-103 suppresses the proliferation and invasion of glioma cells.

$B D N F$ is a direct target of miR-103 in glioma. Potential targets of miR-103 in glioma cells were investigated to elucidate the molecular mechanisms underlying miR-103-mediated inhibition of proliferation and invasion of glioma cells. Bioinformatics analysis was performed to predict candidate targets of miR-103. The analysis indicated a potentially complementary region between the seed region sequence of miR-103 and nucleotide 299-305 region in the 3'-UTR of BDNF (Fig. 3A). To confirm this specificity, regulatory effects of miR-103 on the endogenous BDNF expression were investigated. RT-qPCR and western blot analysis revealed that miR-103 overexpression suppressed BDNF expression in U251 cells at both mRNA and protein levels (Fig. 3B and C respectively, both $\mathrm{P}<0.05$ ). Luciferase reporter assay was conducted to further identify the direct interaction between miR-103 and the 3'-UTR of BDNF. Luciferase activity was decreased in the U251 cells co-transfected with the pmirGLO-BDNF-3'-UTR 
A
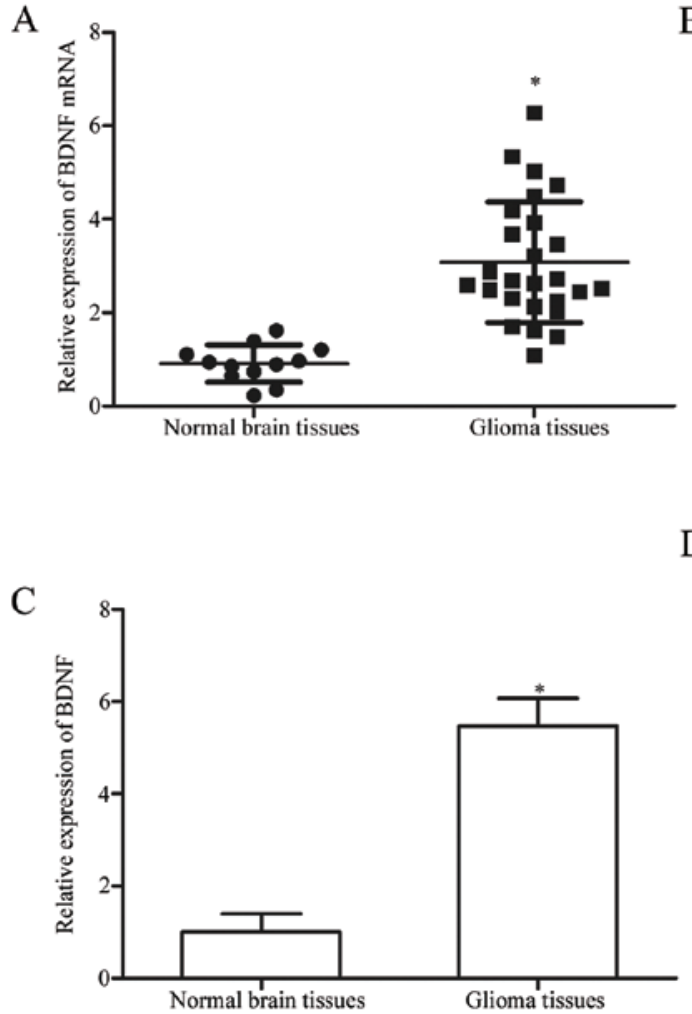

$\mathrm{D}$

B
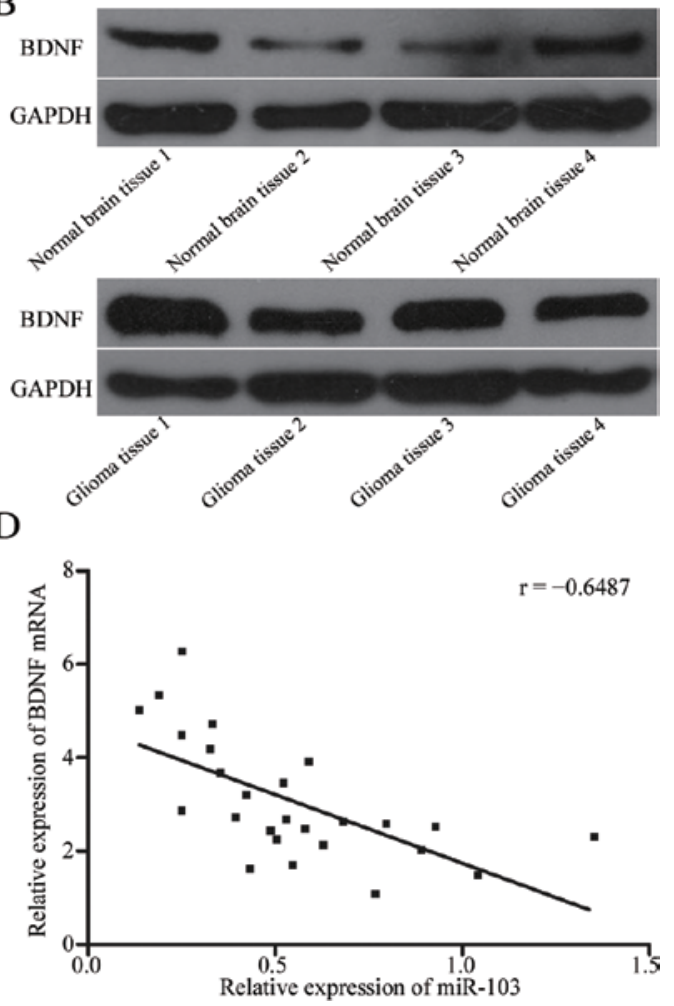

Figure 4. BDNF is upregulated in glioma tissues and is negatively associated with miR-103 expression. BDNF (A) mRNA and (B and C) protein expression was detected in glioma tissues and normal brain tissues. (D) Spearman's correlation analysis was used to investigate the association between BDNF and miR-103 expression level in glioma tissues. Data are presented as the mean \pm standard deviation. " $\mathrm{P}<0.05$ vs. the respective control. miR-103, micoroRNA-103; BDNF, brain-derived neurotrophic factor.

Wt vector and miR-103 mimics (Fig. 3D, P<0.05). The pmirGLO-BDNF-3'-UTR Mut vector along with the miR-103 mimic exerted no effect on luciferase activities. Above data suggest that BDNF is a direct target of miR-103 in glioma.

miR-103 expression is negatively associated with BDNF levels in glioma tissues. mRNA and protein levels of BDNF in glioma tissues and normal brain tissues were measured using RT-qPCR and western blotting, respectively to verify the association between the expression of miR-103 and BDNF. The results demonstrated that mRNA and protein levels of BDNF were significantly increased in glioma tissues compared with normal brain tissues (Fig. 4A-C, both $\mathrm{P}<0.05$ ).

Spearman's correlation analysis demonstrated a negative association between miR-103 and BDNF mRNA expression levels in glioma tissues (Fig. 4D; $r=-0.6487 ; \mathrm{P}=0.0003$ ). The above results suggest that miR-103 down-regulation may cause BDNF up-regulation in glioma.

$B D N F$ overexpression counteracts the suppressive effects of miR-103 on glioma cells. Considering that BDNF is a direct target of miR-103, it was investigated whether BDNF is involved in the regulation of miR-103-mediated inhibition of glioma cell proliferation and invasion. U251 cells were transfected with miR-103 mimics and pcDNA3.1 or pcDNA3.1-BDNF. Western blot analysis indicated that BDNF down-regulation induced by miR-103 was counteracted by BDNF overexpression (Fig. 5A, $\mathrm{P}<0.05$ ). Functional rescue experiments revealed that BDNF up-regulation significantly decreased the suppressive effects of miR-103 on glioma cell proliferation and invasion (Fig. 5B and $\mathrm{C}$; both $\mathrm{P}<0.05$ ). The above results suggest that miR-103 may inhibit the proliferation and invasion of glioma cells by suppressing BDNF expression.

\section{Discussion}

miRNAs are involved in the tumour development of various human cancers, including glioma (29-31). Dysregulation of miRNAs is a common feature of human malignancies, as they regulate the expression of oncogenes and tumour suppressors (32). Therefore, miRNAs may possess potential tumour diagnostic, prognostic and therapeutic values in human glioma. In the present study, miR-103 was down-regulated in glioma tissues and cell lines. In addition, restoration of normal-cell levels of expression of miR-103 attenuated glioma cell proliferation and invasion in vitro. Furthermore, BDNF was validated as a direct target of miR-103 in glioma. The above results indicate that miR-103 acts as a tumour suppressor in glioma, suggesting its potential use in the treatment of patients with glioma.

miR-103 is down-regulated in several human cancers, including gastric cancer tissues and cell lines. Low miR-103 expression is associated with pathological (p)M stage in the tumor, node and metastasis classification of patients with gastric cancer (33).In the prostate cancer, miR-103 expression is reduced in tumour tissues (27). However, miR-103 is overexpressed in colorectal cancer. High miR-103 expression is associated with 
A

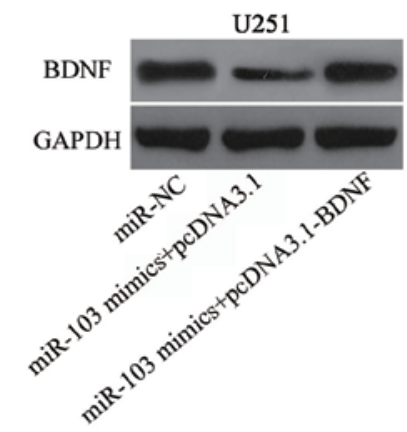

B

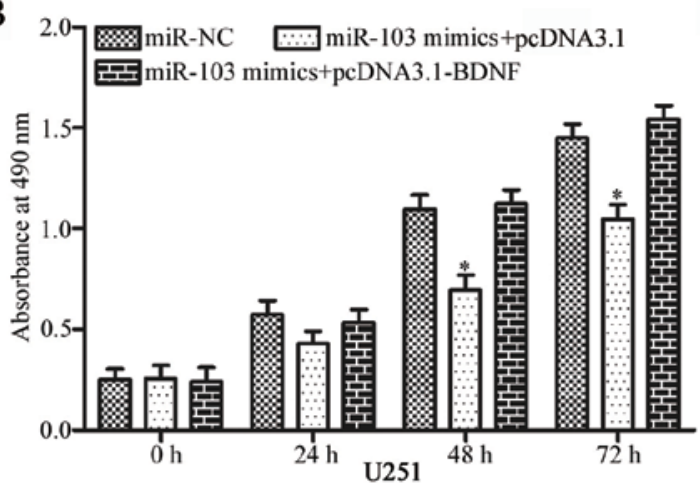

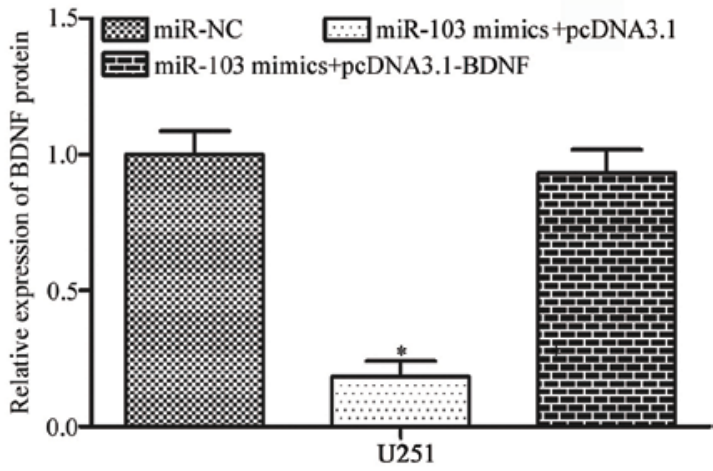

$\mathrm{C}$

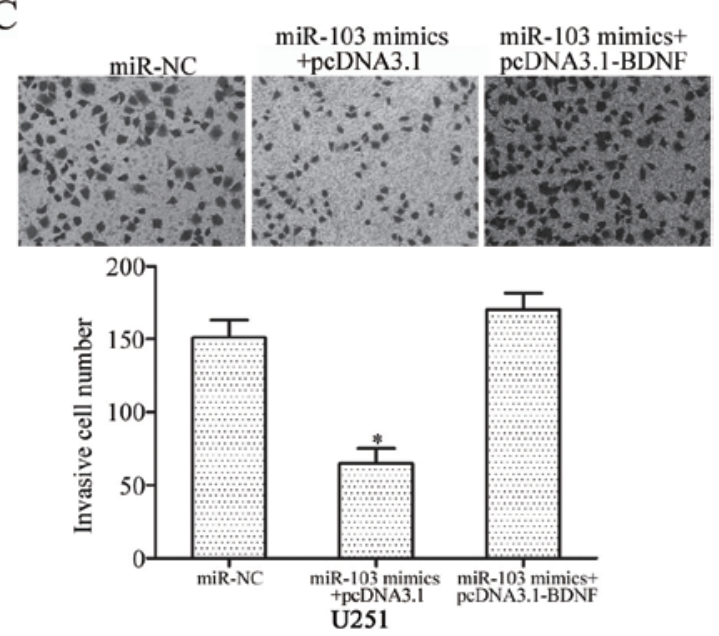

Figure 5. BDNF up-regulation reverses the inhibitory effects of miR-103 on the proliferation and invasion of glioma cells. (A) BDNF protein expression was measured in U251 cells co-transfected with miR-103 mimics with pcDNA3.1 or pcDNA3.1-BDNF. (B) MTT assay was used to detect the proliferation of U251 cells co-transfected with miR-103 mimics with pcDNA3.1 or pcDNA3.1-BDNF. (C) Transwell invasion assay was used to detect the invasion capacity of U251 cells co-transfected with miR-103 mimics pcDNA3.1 or pcDNA3.1-BDNF. Data are presented as the mean \pm standard deviation. ${ }^{*} \mathrm{P}<0.05$ vs. the respective control. miR-103, micoroRNA-103; BDNF, brain-derived neurotrophic factor; NC, negative control.

an aggressive phenotype of colorectal cancer and poor overall survival of patients (34). miR-103 is also up-regulated in breast cancer and hepatocellular carcinoma $(35,36)$. Serum levels of miR-103 in breast cancer are associated with the advanced clinical stage and lymph node metastasis (35). The above results indicate that the expression patterns of miR-103 are tissue specific and can be used as a predictive value for patient prognosis in human cancer.

miR-103 has been previously identified to serve tumour-suppressing roles in multiple human malignancies. For instance, miR-103 up-regulation suppresses cell growth and metastasis in gastric cancer (33). Restoration of the expression of miR-103 inhibits prostate cancer cell proliferation and invasion and increases the duration of the G1 phase of the cell cycle (27). miR-103 was also identified as an oncogene in colorectal cancer, promoting cell growth and metastasis both in vitro and in vivo $(25,34)$. Yu et al $(26)$ revealed that miR-103 downregulation attenuates cell proliferation and invasion of endometrial cancer. Xia et al (36) reported that miR-103 overexpression promotes cell proliferation and decreases apoptosis in hepatocellular carcinoma. The above, conflicting results revealed that the biological functions of miR-103 are tissue specific, an observation which can be explained by imperfect complementarity between miRNAs and their target genes. Therefore, miR-103 should be investigated as a potential target for the therapeutic treatment of specific types of cancer.
Previous studies have identified several miR-103 targets, including: i) c-Myb proto-oncogene in gastric cancer; ii) large tumor suppressor kinase 2, endoribonuclease DICER and phosphatase and tensin homolog in colorectal cancer; iii) tissue inhibitor of metallopeptidases- 3 in endometrial cancer; iv) programmed cell death 10 in prostate cancer; and v) A-kinase anchoring protein 12 in hepatocellular carcinoma $(25-27,33,34,36)$. In the present study, BDNF was validated as a novel target of miR-103. A bioinformatics prediction indicated that BDNF is a potential target of miR-103. RT-qPCR and western blotting demonstrated that miR-103 negatively modulated the mRNA and protein expression levels of BDNF in glioma cells. Luciferase reporter assay revealed that the 3'-UTR of BDNF could be directly targeted by miR-103 in glioma cells. Furthermore, BDNF was significantly upregulated in glioma tissues and negatively associated with miR-103 expression. Rescue experiments demonstrated that BDNF overexpression substantially reversed the suppressive effects of miR-103 on glioma cell proliferation and invasion. The above results indicate that BDNF is a direct and functional target gene of miR-103 in glioma.

BDNF, located on the short arm of chromosome 11 (11p13), is up-regulated in various human cancer types, including gastric, lung, cervical, breast and colorectal cancers, and hepatocellular carcinoma (37-42). BDNF has been recently identified to act as a cancer regulator and serve a role in cancer occurrence and 
development (43). In glioma, BDNF is highly expressed in tumour tissues and is associated with high pathological grading scores (44). Functional assays indicated that BDNF is an oncogene in glioma as it promotes cell growth and metastasis, and inhibits apoptosis (45). Therefore, BDNF may be a promising therapeutic target for the treatment of patients with glioma.

In conclusion, the present study provided evidence that miR-103 is down-regulated in glioma tissues and cell lines. miR-103 serves a tumour-suppressive role in glioma by down-regulating BDNF. The results of the present study indicate that the miR-103/BDNF interaction is a potential therapeutic target for the treatment of glioma. However, the use of a single cell line is a limitation of the present study, and the results should be validated using multiple cell lines.

\section{References}

1. Goodenberger ML and Jenkins RB: Genetics of adult glioma. Cancer Genet 205: 613-621, 2012.

2. Buckner JC, Brown PD, O'Neill BP, Meyer FB, Wetmore CJ and Uhm JH: Central nervous system tumors. Mayo Clin Proc 82: 1271-1286, 2007.

3. Liu X, Wang S, Yuan A, Yuan X and Liu B: MicroRNA-140 represses glioma growth and metastasis by directly targeting ADAM9. Oncol Rep 36: 2329-2338, 2016.

4. Zhu GY, Shi BZ and Li Y: FoxM1 regulates Sirt1 expression in glioma cells. Eur Rev Med Pharmacol Sci 18: 205-211, 2014.

5. Chen J, McKay RM and Parada LF: Malignant glioma: Lessons from genomics, mouse models, and stem cells. Cell 149:36-47,2012.

6. Stupp R, Mason WP, van den Bent MJ, Weller M, Fisher B, Taphoorn MJ, Belanger $\mathrm{K}$, Brandes AA, Marosi C, Bogdahn U, et al: Radiotherapy plus concomitant and adjuvant temozolomide for glioblastoma. N Engl J Med 352: 987-996, 2005.

7. Van Meir EG, Hadjipanayis CG, Norden AD, Shu HK, Wen PY and Olson JJ: Exciting new advances in neuro-oncology: The avenue to a cure for malignant glioma. CA Cancer J Clin 60: 166-193, 2010.

8. Lefranc F, Brotchi J and Kiss R: Possible future issues in the treatment of glioblastomas: Special emphasis on cell migration and the resistance of migrating glioblastoma cells to apoptosis. J Clin Oncol 23: 2411-2422, 2005

9. Onishi M, Ichikawa T, Kurozumi K and Date I: Angiogenesis and invasion in glioma. Brain Tumor Pathol 28: 13-24, 2011.

10. Carrington JC and Ambros V: Role of microRNAs in plant and animal development. Science 301: 336-338, 2003.

11. Qin B, Yang H and Xiao B: Role of microRNAs in endothelial inflammation and senescence. Mol Biol Rep 39: 4509-4518, 2012

12. Kotaja N: MicroRNAs and spermatogenesis. Fertil Steril 101: $1552-1562,2014$

13. Imbar T and Eisenberg I: Regulatory role of microRNAs in ovarian function. Fertil Steril 101: 1524-1530, 2014.

14. Garg D and Cohen SM: miRNAs and aging: A genetic perspective. Ageing Res Rev 17: 3-8, 2014.

15. Dogini DB, Pascoal VD, Avansini SH, Vieira AS, Pereira TC and Lopes-Cendes I: The new world of RNAs. Genet Mol Biol 37 (1 Suppl): S285-S293, 2014.

16. Hong L, Ya-Wei L, Hai W, Qiang Z, Jun-Jie L, Huang A, Song-Tao Q and Yun-Tao L: MiR-519a functions as a tumor suppressor in glioma by targeting the oncogenic STAT3 pathway. J Neurooncol 128: 35-45, 2016.

17. Teoh SL and Das S: The role of MicroRNAs in diagnosis, prognosis, metastasis and resistant cases in breast cancer. Curr Pharm Des 23: 1845-1859, 2017.

18. Liu HT, Wang YW, Xing AY, Shi DB, Zhang H, Guo XY, Xu J and Gao P: Prognostic value of microRNA signature in patients with gastric cancers. Sci Rep 7: 42806, 2017.

19. Dong F, Xu T, Shen Y, Zhong S, Chen S, Ding Q and Shen Z: Dysregulation of miRNAs in bladder cancer: Altered expression with aberrant biogenesis procedure. Oncotarget 8: 27547-27568, 2017.

20. Liu L, Bi N, Wu L, Ding X, Men Y, Zhou W, Li L, Zhang W, Shi S, Song Y and Wang L: MicroRNA-29c functions as a tumor suppressor by targeting VEGFA in lung adenocarcinoma. Mol Cancer 16: 50, 2017.
21. Chen Y, Gao DY and Huang L: In vivo delivery of miRNAs for cancer therapy: Challenges and strategies. Adv Drug Deliv Rev 81: 128-141, 2015.

22. Turner JD, Williamson R, Almefty KK, Nakaji P, Porter R, Tse V and Kalani MY: The many roles of microRNAs in brain tumor biology. Neurosurg Focus 28: E3, 2010.

23. Bartel DP: MicroRNAs: Genomics, biogenesis, mechanism, and function. Cell 116: 281-297, 2004.

24. Kong YW, Ferland-McCollough D, Jackson TJ and Bushell M: microRNAs in cancer management. Lancet Oncol 13: e249-e258, 2012.

25. Geng L, Sun B, Gao B, Wang Z, Quan C, Wei F and Fang XD: MicroRNA-103 promotes colorectal cancer by targeting tumor suppressor DICER and PTEN. Int J Mol Sci 15: 8458-8472, 2014.

26. Yu D, Zhou H, Xun Q, Xu X, Ling J and Hu Y: microRNA-103 regulates the growth and invasion of endometrial cancer cells through the downregulation of tissue inhibitor of metalloproteinase 3. Oncol Lett 3: 1221-1226, 2012.

27. Fu X, Zhang W, Su Y, Lu L, Wang D and Wang H: MicroRNA-103 suppresses tumor cell proliferation by targeting PDCD10 in prostate cancer. Prostate 76: 543-551, 2016.

28. Livak KJ and Schmittgen TD: Analysis of relative gene expression data using real-time quantitative PCR and the 2(-Delta Delta C(T)) method. Methods 25: 402-408, 2001

29. Neviani P and Fabbri M: Exosomic microRNAs in the tumor microenvironment. Front Med (Lausanne) 2: 47, 2015

30. Zhao Y, Pang D, Wang C, Zhong S and Wang S: MicroRNA-134 modulates glioma cell U251 proliferation and invasion by targeting KRAS and suppressing the ERK pathway. Tumour Biol 37: 11485-11493, 2016.

31. Tian LQ, Liu EQ, Zhu XD, Wang XG, Li J and Xu GM: MicroRNA-197 inhibits cell proliferation by targeting GAB2 in glioblastoma. Mol Med Rep 13: 4279-4288, 2016.

32. Garzon R, Calin GA and Croce CM: MicroRNAs in Cancer. Annu Rev Med 60: 167-179, 2009.

33. Liang J, Liu X, Xue H, Qiu B, Wei B and Sun K: MicroRNA-103a inhibits gastric cancer cell proliferation, migration and invasion by targeting c-Myb. Cell Prolif 48: 78-85, 2015.

34. Zheng YB, Xiao K, Xiao GC, Tong SL, Ding Y, Wang QS, Li SB and Hao ZN: MicroRNA-103 promotes tumor growth and metastasis in colorectal cancer by directly targeting LATS 2 . Oncol Lett 12: 2194-2200, 2016.

35. Wang X, Wu X, Yan L and Shao J: Serum miR-103 as a potential diagnostic biomarker for breast cancer. Nan Fang Yi Ke Da Xue Xue Bao 32: 631-634, 2012 (In Chinese).

36. Xia W, Ni J, Zhuang J, Qian L, Wang P and Wang J: MiR-103 regulates hepatocellular carcinoma growth by targeting AKAP12. Int J Biochem Cell Biol 71: 1-11, 2016.

37. Choi B, Lee EJ, Shin MK, Park YS, Ryu MH, Kim SM, Kim EY, Lee HK and Chang EJ: Upregulation of brain-derived neurotrophic factor in advanced gastric cancer contributes to bone metastatic osteolysis by inducing long pentraxin 3 . Oncotarget 7: 55506-55517, 2016

38. Zhang SY, Hui LP, Li CY, Gao J, Cui ZS and Qiu XS: More expression of BDNF associates with lung squamous cell carcinoma and is critical to the proliferation and invasion of lung cancer cells. BMC Cancer 16: 171, 2016.

39. Moon A, Won KY, Lee JY, Kang I, Lee SK and Lee J: Expression of BDNF, TrkB, and p53 in early-stage squamous cell carcinoma of the uterine cervix. Pathology 43: 453-458, 2011.

40. Patani N, Jiang WG and Mokbel K: Brain-derived neurotrophic factor expression predicts adverse pathological \& clinical outcomes in human breast cancer. Cancer Cell Int 11: 23, 2011.

41. Yang X, Martin TA and Jiang WG: Biological influence of brain-derived neurotrophic factor (BDNF) on colon cancer cells. Exp Ther Med 6: 1475-1481, 2013.

42. Yang ZF, Ho DW, Lam CT, Luk JM, Lum CT, Yu WC, Poon RT and Fan ST: Identification of brain-derived neurotrophic factor as a novel functional protein in hepatocellular carcinoma. Cancer Res 65: 219-225, 2005

43. Tajbakhsh A,Mokhtari-Zaer A, Rezaee M, Afzaljavan F, Rivandi M, Hassanian SM, Ferns GA, Pasdar A and Avan A: Therapeutic potentials of BDNF/TrkB in breast cancer; current status and perspectives. J Cell Biochem 118: 2502-2515, 2017.

44. Yan Q, Yu HL and Li JT: Study on the expression of BDNF in human gliomas. Sichuan Da Xue Xue Bao Yi Xue Ban 40: 415-417, 2009 (In Chinese).

45. Xiong J, Zhou L, Lim Y, Yang M, Zhu YH, Li ZW, Zhou FH, Xiao ZC and Zhou XF: Mature BDNF promotes the growth of glioma cells in vitro. Oncol Rep 30: 2719-2724, 2013. 